\title{
Context fear learning specifically activates distinct populations of neurons in amygdala and hypothalamus
}

\author{
Lidia Trogrlic, ${ }^{1}$ Yvette M. Wilson, ${ }^{1}$ Andrew G. Newman, and Mark Murphy ${ }^{2}$ \\ Department of Anatomy and Cell Biology, University of Melbourne, Melbourne, Victoria 3010, Australia
}

\begin{abstract}
The identity and distribution of neurons that are involved in any learning or memory event is not known. In previous studies, we identified a discrete population of neurons in the lateral amygdala that show learning-specific activation of a c-fos-regulated transgene following context fear conditioning. Here, we have extended these studies to look throughout the amygdala for learning-specific activation. We identified two further neuronal populations, in the amygdalo-striatal transition area and medial amygdala, that show learning-specific activation. We also identified a population of hypothalamic neurons that show strong learning-specific activation. In addition, we asked whether these neurons are activated following recall of fear-conditioning memory. None of the populations of neurons we identified showed significant memory-recallrelated activation. These findings suggest that a series of discrete populations of neurons are involved in fear learning in amygdala and hypothalamus. The lack of reactivation during memory recall suggests that these neurons either do not undergo substantial change following recall, or that c-fos is not involved in any such activation and change.
\end{abstract}

Pavlovian fear conditioning is one of the best-characterized models for the study of learning and memory and its neural correlates. It involves the association of a neutral stimulus, the conditioned stimulus (CS) such as a sound or context, with an aversive unconditioned stimulus (US) such as a footshock. Fear conditioning is quite robust, and a large percentage of trained animals show fear learning. It can be examined with respect to several excellent nonlearning controls. Some of the major areas of the brain that are necessary for expression of fear conditioning have been determined by lesioning experiments, with the amygdala playing a central role in the process (Pitkanen et al. 1997; LeDoux 2000; Sah et al. 2003; Fanselow and Poulos 2005; Ehrlich et al. 2009).

A large body of evidence implicates the lateral amygdala in the formation and storage of fear memories in the amygdala (LeDoux 1993; Fanselow and LeDoux 1999; Maren and Quirk 2004; Pare et al. 2004; Rodrigues et al. 2004); however, this region does not act in isolation. Anterograde and retrograde tracing studies have yielded a plethora of amygdalar efferents and afferents. The amygdala itself is comprised of several heavily interconnected nuclei and receives a massive array of both cortical (McDonald 1998) and subcortical inputs (Pitkanen et al. 1997; Sah et al. 2003). The amygdala also receives modulatory monoaminergic input from hypothalamic nuclei (Asan et al. 2005); however, this remains poorly characterized.

In moving beyond basic functional neuroanatomy, progress in our understanding of the brain requires not only a comprehension of the mechanisms of plasticity, but also of the localized circuitry involved in specific behaviors. Our previous development of the transgenic fos-tau-lacZ (FTL) mouse (Wilson et al. 2002; Murphy et al. 2007) has emerged as an excellent tool for visualizing functionally activated early gene expression in brain subnuclei associated with learning and memory. Here, we sought to expand upon the initial finding that a distinct population of cells in the lateral amygdala (LA) is activated by fear learning (Wilson and Murphy 2009). An examination of incidental activation of

\footnotetext{
${ }^{1}$ These authors contributed equally to this work.

${ }^{2}$ Corresponding author.

E-mail m.murphy@unimelb.edu.au.

Article is online at http://www.learnmem.org/cgi/doi/10.1101//m.2314311.
}

other brain nuclei should provide insight into the regions involved in fear-learning circuitry.

The present study has examined all amygdala subnuclei to look for learning-specific activation in the FTL mice. We have compared FTL activation following fear-conditioning training, with activation seen following a series of different sensory controls as well as following fear-memory recall. Using this multicomparison approach, we have sought to distinguish learning-specific activation from nonlearning-related activation such as that related to sensory input, fear expression, and memory recall. We identify a series of different amygdala nuclei in addition to a region in the hypothalamus that shows activation patterns specific to fear learning.

\section{Results}

Behavioral analysis of fear conditioning

We undertook context fear conditioning of FTL mice in order to identify brain regions that were activated by fear learning and recall of fear memory. Two sets of behavioral experiments were undertaken. The first set involved training the mice in fear conditioning (the training set), then analyzing FTL activation after $4 \mathrm{~h}$. FTL expression here is due to training. The second set of experiments involved training the mice on fear conditioning, testing the mice $4 \mathrm{~d}$ later for recall of fear memory (the recall set), and then analyzing FTL activation $4 \mathrm{~h}$ later. FTL expression here is due to testing or recall.

In the training set, the conditioned (learning) group was placed in a shock chamber and received a small shock after $3 \mathrm{~min}$. We used a number of different control groups in order to distinguish between FTL activation due to context fear learning and FTL activation due to nonlearning experiences. To control for a simple sensory experience, we used a context group that received no shock and an immediate shock group. To control for the two serial novel stimuli (context, then shock), which the conditioned mice received, we used two further groups. The first was a tone control, wherein the mice received an auditory stimulus in place of a shock 3 min after being placed in the shock chamber. This control is thus a presentation of two novel serial stimuli, 
but without conditioning. The second involves a context preexposure $1 \mathrm{~d}$ prior to fear conditioning. The context pre-exposure makes the context familiar when the mice are conditioned, and thus effectively means the mice only receive one novel stimulus during conditioning.

Neither context, immediate shock, or tone mice displayed any significant freezing behavior during training or testing, denoting no fear conditioning to the context (Fig. 1A). The tone mice showed a small decrease in movement following the presentation of the tone, but there was no significant decrease at testing (Fig. 1B). Conditioned mice showed a clear freezing response. The conditioned group froze significantly more both during the post-shock interval $(P<0.05)$ and when placed back into the chamber for testing $(P<0.001)$ compared with pre-shock $\left(F_{(2,23)}=12.28\right)$. Conditioned mice also showed significant decreases in movement during post-shock $(P<0.001)$ and testing $(P<0.001)$ time intervals compared with pre-shock $\left(F_{(2,24)}=\right.$ 48.18; Fig. 1B). Mice that were pre-exposed to the context prior to being conditioned showed a very similar pattern of responses to the conditioned mice (Fig. 1A,B), but showed higher freezing responses on testing $\left(P<0.05 ; F_{(5,44)}=10.13\right.$; Fig. $\left.1 \mathrm{~A}\right)$. These findings demonstrate that only the conditioned groups acquired fear learning and memory.

In the recall set, we used two groups: a group that had previously been trained to acquire fear conditioning using the conditioned protocol described above (conditioned recall group) and a group that was exposed only to context in the initial training (context recall group). Again, in this set only the conditioned recall group of mice exhibited behavior consistent with context fear conditioning (Fig. 2A,B). The analysis of freezing response during training and testing revealed that conditioned recall mice froze significantly more at testing for memory recall than during the pre-shock training time interval $\left(F_{(2,22)}=9.417 ; P<\right.$ 0.001; Fig. 2A) and moved significantly less post-shock and at testing compared with pre-shock $\left(F_{(2,22)}=130.3 ; P<0.001\right.$; Fig. $\left.2 \mathrm{~B}\right)$. There were no effects on freezing or moving behavior in the context recall group (Fig. 2A,B).

\section{Analysis of FTL expression in amygdala following fear-conditioning training and following recall}

\section{Lateral amygdala-ventrolateral region}

Our previous work identified a specific population of neurons in the amygdala that express FTL following context fear learning (Wilson and Murphy 2009). This population of neurons was in the ventrolateral region (vl) of the lateral amygdala (LA). These learning-specific neurons were also found only in mid to posterior levels of the LA (Bregma -1.78 to $-1.98 \mathrm{~mm}$ ). In order to determine whether a similar learning-specific population was activated in the present experiment, the LA was examined for each behavioral group of both the training set as well as the recall set (Figs. 3, 4). A significant increase in the number of $F T L^{+}$cells was observed in the conditioned groups compared with all other groups $\left(F_{(8,31)}=32.56\right.$; Fig. 4$)$. In addition, the pre-expose conditioned group showed a further increase in $F T L^{+}$neuron number compared with the conditioned group $(P<0.01)$. There were apparent increases in $\mathrm{FTL}^{+}$neurons in context, immediate shock, and tone groups compared with home cage, but these were not significant (Figs. 3, 4). The $\mathrm{FTL}^{+}$cells in the LAvl from the trained mice had the same multiprocess stellate appearance and large diameter (Fig. 3G) as those described in our previous study (Wilson and Murphy 2009).

For the analysis of $F T L$ expression during recall, an additional control group was added. This group, conditioned recall $0 \mathrm{~h}$, had the same training and testing as the conditioned recall group,
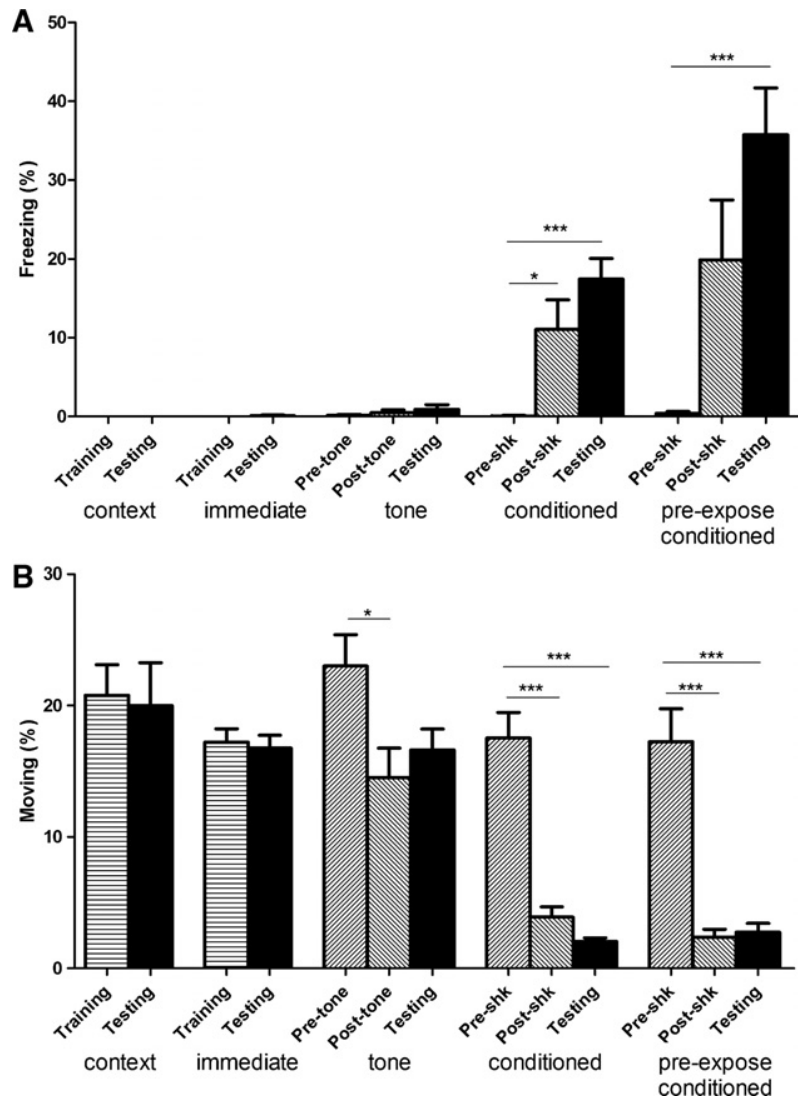

Figure 1. Contextual fear conditioning of FTL mice. FTL mice were placed in the training chamber and either received no shock (context), were shocked immediately (immediate group), received a tone (tone), or received a shock after 3 min (conditioned). Pre-expose conditioned mice were placed in the chamber for $3 \mathrm{~min} 1 \mathrm{~d}$ prior to being conditioned. At the end of training, all mice were removed and placed in their home cages. Four hours later the mice were placed back into the training chamber for $3 \mathrm{~min}$ for testing. Fear response was tested by measuring freezing $(A)$ and moving time $(B)$ during the whole training period for the context and immediate shock groups, during the pre-tone and posttone period for the tone mice, and the pre-shock and post-shock period for the conditioned groups; all mice were also measured during the testing period. Shown are mean \pm SEM for each group. $\left(^{*}\right) P<0.05$; $(* * *) P<0.001$.

except the mice were killed immediately after testing for recall. In this group, there can be no FTL expression due to testing. This group was included to account for any FTL expression that could have resulted from the initial fear learning $4 \mathrm{~d}$ previously. There were no significant differences in the number of $F T L^{+}$neurons within the recall set or compared with the home-cage group (Figs. 3, 4). The only significant increases in $F L^{+}$neuron number over any other group were in the conditioned groups (Fig. 4). These findings suggest that c-fos-related activation of the FTL transgene within the LAvl is specifically related to fear learning.

\section{Amygdalo-striatal transition area}

The amygdalo-striatal transition area (AStr) also showed learning-specific differences in FTL expression. This population of neurons is located between the central amygdala and the LA (Fig. 5) and from Bregma -1.06 to $-1.36 \mathrm{~mm}$ (Franklin and Paxinos 2007). As in the LAvl, analysis was conducted of the training and recall sets. In the training set, there were significant increases in the number of $F_{T L}^{+}$neurons in both conditioned 

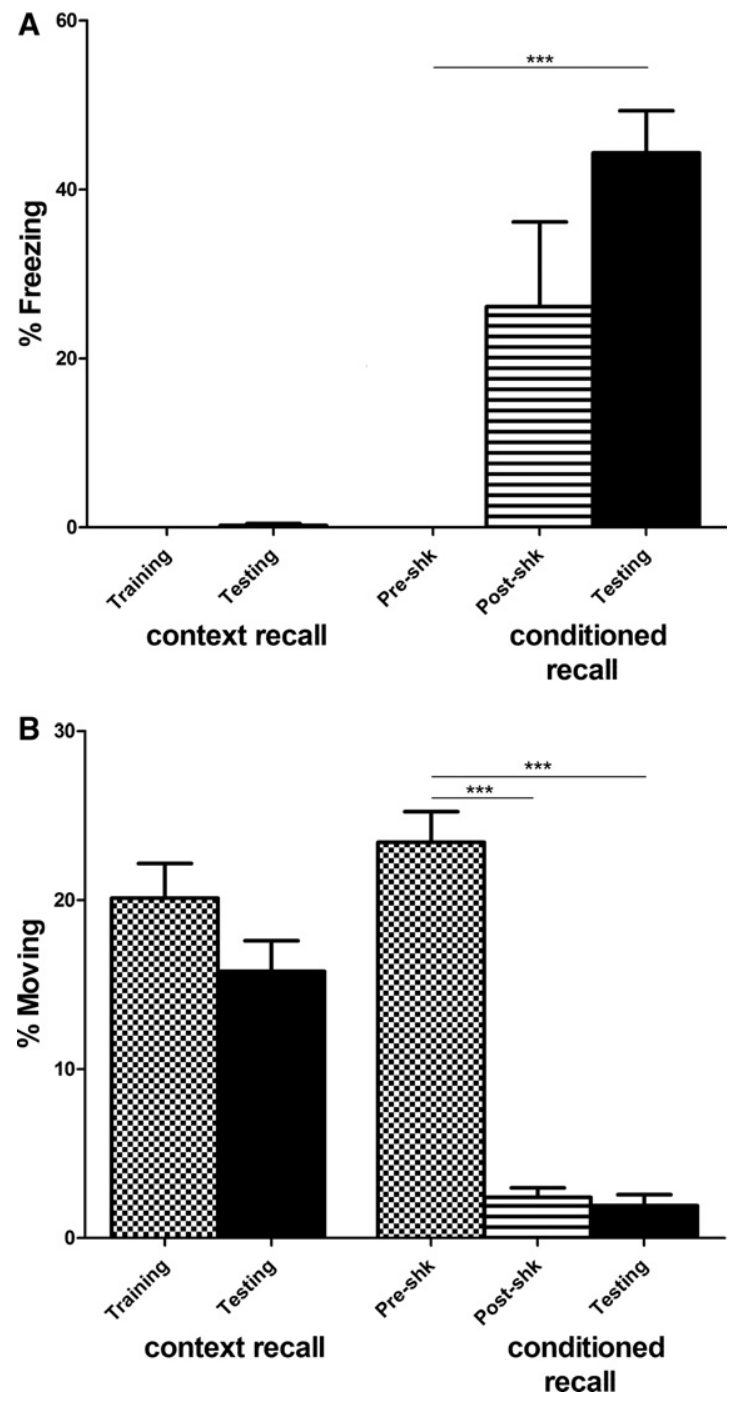

Figure 2. Contextual fear recall of $F T L$ mice. FTL mice were placed in the training chamber and either received no shock (context recall) or shocked after 3 min (conditioned recall). At the end of training, all mice were removed and placed in their home cages. Four days later the mice were placed back into the training chamber for 3 min for testing. Fear response was tested by measuring freezing $(A)$ and moving time $(B)$ during the testing period. Shown are mean \pm SEM for each group. ${ }^{* * *}$ $P<0.001$.

groups compared with all other groups $\left(F_{(8,29)}=22.86\right.$; Fig. 6A) The increases represented an approximately fourfold increase compared with the home-cage group, and an approximately twofold increase compared with context, immediate shock, or tone groups. There was also a significant increase in the numbers of $\mathrm{FTL}^{+}$neurons in context, immediate shock, and tone groups (Fig. 5B,C) compared with the home cage $(P<0.05)$ and conditioned recall 0 -h group $(P<0.01$; Fig. 6A).

Within the recall set there were small, but insignificant trends suggesting an increase in $F T L^{+}$neuron number following both context recall and conditioned recall (Fig. 6A). In summary, these findings suggest that FTL activation within the AStr is predominantly associated with fear learning.

FTL staining within this region from the conditioned mice highlighted the morphology of these cells (Fig. 5G). The neurons appeared to be fairly heterogeneous in size and appearance, including many small neurons. The diameter of the neurons ranged from 6.7 to $15 \mu \mathrm{m}$, with an overall average of $9.8 \pm$ $1.4 \mu \mathrm{m}(n=177)$. The size distribution did not fall into discrete classes, but it was also not normally distributed. Possibly, more than one neuronal type was present.

\section{Medial amygdala}

The medial amygdala (MeA) was the third amygdala region to show learning-related changes in FTL expression (Fig. 5). The learning-specific neurons were located between Bregma -1.16 and $-1.36 \mathrm{~mm}$ (Franklin and Paxinos 2007). The changes in the MeA showed a trend similar to that seen for both LAvl and AStr, with the conditioned groups showing highly significant increases in $F_{T L}^{+}$neurons compared with all other groups $\left(F_{(8,29)}=21.36\right.$; Fig. 6B). The increase in the number of $\mathrm{FTL}^{+}$neurons in the conditioned group was approximately fourfold compared with home cage, and conditioned recall 0 -h controls and approximately twofold compared with the other groups (Fig. 6B). The pre-expose conditioned group had a further increase in neuron number compared with the conditioned group $(P<0.05$; Fig. 6B). These findings suggest that the main effect on FTL activation within MeA in these experiments is specifically associated with fear learning. There was a considerable variation in appearance of the $\mathrm{FTL}^{+}$neurons within the MeA (Fig. $5 \mathrm{H}$ ), similar to that seen in the AStr. Possibly, this variation was due to different classes of neurons being activated during learning. The mean diameter of the neurons was $11.45 \pm 2.27 \mu \mathrm{m}(n=168)$.

\section{Hypothalamus}

A region within the hypothalamus that appeared to show strong learning-associated changes in FTL expression was also studied (Fig. 7). The learning-related changes were within the region of the anterior and ventromedial hypothalamus (VMH), and Bregma -1.04 to $-1.44 \mathrm{~mm}$. By comparison with the Mouse Brain Atlas (Franklin and Paxinos 2007), the activated neurons appeared to mainly fall within the $\mathrm{VMH}$, but also extended into the posterior part of the anterior hypothalamic area (Fig. 7).

The conditioned groups had increased numbers of $\mathrm{FTL}^{+}$neurons compared with the other groups in the $\operatorname{VMH}\left(F_{(8,30)}=26.29\right.$; $P<0.001$; Fig. 8). This increase in neuron number was at least threefold compared with all other groups. The pre-expose conditioned group had a further increase in neuron number compared with the conditioned group $(P<0.01 ;$ Fig. 8$)$. There were no other significant changes (Figs. 7, 8). The learning-specific cells of this region generally appeared to be large, densely stained neurons (diameter $14.77 \pm 2.86 \mu \mathrm{m}[n=143]$; Fig. $7 \mathrm{G}$ ).

\section{Discussion}

The objective of this study was to identify populations of neurons that are specifically activated in context fear learning and to determine whether these same populations were activated following fear-memory recall. For this purpose, we used FTL mice to visualize functionally activated early gene expression in neurons, which use the c-fos promoter. Induction of c-fos not only requires depolarization and increases in firing rate, but is also associated with strong activation of neurotransmitter receptors and substantial changes in intracellular $\mathrm{Ca}^{++}$(Cirelli and Tononi 2000; Kovacs 2008). Thus, c-fos activation is considered to be indicative of strong activation of cell functioning during periods of plasticity or high rates of metabolic activity, and not simply a marker of neuronal firing (Cirelli and Tononi 2000; Kovacs 2008).

Initially, only the amygdala was studied, but intense learning-specific FTL expression was obvious within the hypothalamus, and thus, this brain area was also included in the analysis. 

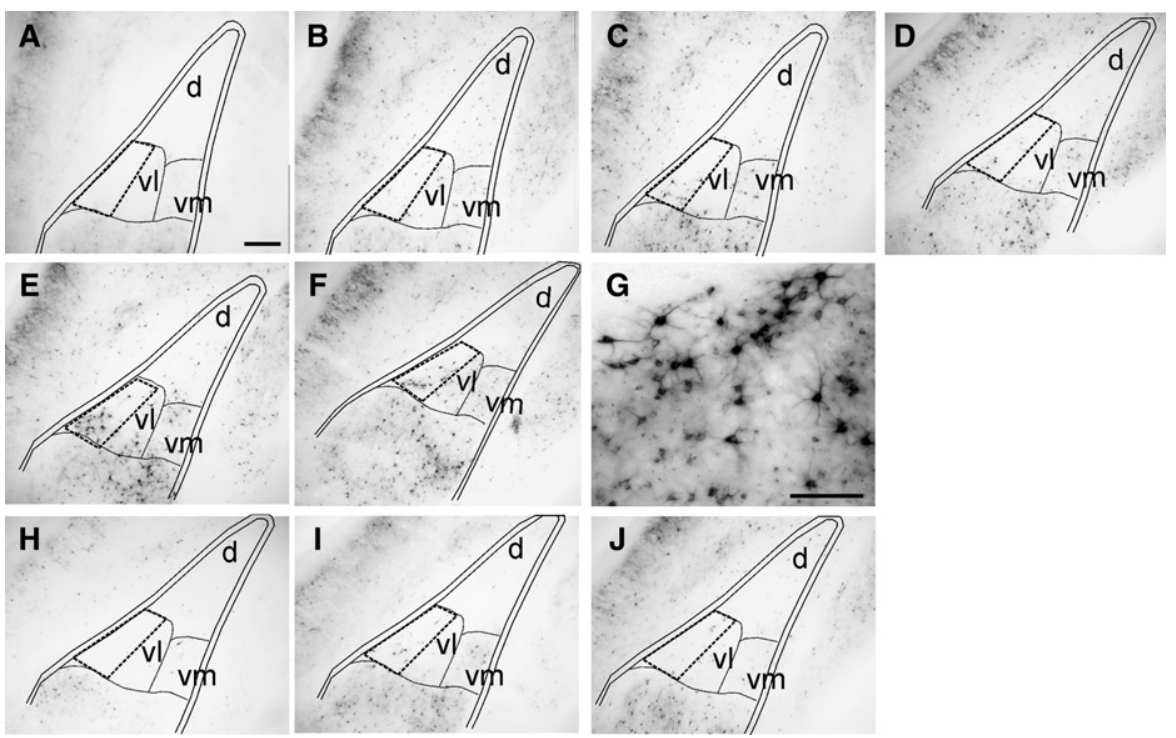

Figure 3. Learning-specific FTL activation in lateral amygdala. Images of mid-posterior LA are shown for FTL mice from the following training groups: $(A)$ home cage, $(B)$ context, $(C)$ immediate shock, $(D)$ tone, $(E)$ conditioned, $(F)$ pre-expose conditioned. $(G)$ A high-power view of $F T L^{+}$neurons within the area of the LAvl that was counted from a pre-exposed conditioned mouse. Images of LA are shown from the following recall groups: $(H)$ conditioned recall $0 \mathrm{~h},(I)$ context recall, and $(J)$ conditioned recall $4 \mathrm{~h}$. The rectangle represents the area within LAvl in which $F T L^{+}$neurons were counted for each condition. The LA subnuclei are outlined and indicated: (d) dorsal; (vl) ventrolateral; (vm) ventromedial. Scale bar: $A-F, H-J=200 \mu \mathrm{m} ; G=100 \mu \mathrm{m}$.

Increases in the number of $\mathrm{FTL}^{+}$neurons were found in LAvl, AStr, $\mathrm{MeA}$, and $\mathrm{VMH}$ following fear learning. We undertook an extensive range of behavioral tests to more completely control for FTL activation due to nonlearning experiences. The inclusion of these tests enabled us to control for expression due to context, shock, or two serial novel stimuli, which the mice receive during normal conditioning. Further, our tests for recall reveal expression not only due to recall of fear memory, but also to the expression of fear, which the mice express following both recall and fear conditioning training. Little increase in numbers of $\mathrm{FTL}^{+}$neurons were found in LAvl, AStr, MeA, and VMH following these control tests. There was also little FTL activation in these regions following recall of fear memory and the expression of fear. These data thus support the argument that the neurons we identified are specifically activated by fear learning.

It should be noted that we have only investigated areas that show c-fos activation. There may be areas within amygdala and hypothalamus that show learning-specific decreases in c-fos expression. For example, other studies show that conditioned fear results in decreased c-fos expression in central amygdala (Day et al. 2008). Such decreases in c-fos expression suggest that these areas were specifically inhibited, as shown by the studies of Day et al. $(2005,2008)$, who correlated inhibition of central amygdala by unconditioned stressors with decreased c-fos expression in the same area following conditioned fear.

\section{Learning-specific neurons in the LAvl}

Our previous studies identified a discrete population of neurons within the LAvl that were specifically activated following context fear learning (Wilson and Murphy 2009). Here, we confirm and extend these findings. In our current fear-conditioning experiments, we find learning-specific FTL expression in the same region of the LAvl as previously reported (Wilson and Murphy 2009). Other studies have reported c-fos or other immediate early gene expression in LA following fear conditioning or footshock (Radulovic et al. 1998; Rosen et al. 1998; Savonenko et al. 1999;
Schettino and Otto 2001; Radwanska et al. 2002; Majak and Pitkanen 2003; Holahan and White 2004; Knapska et al. 2007; Lanuza et al. 2008; Furlong et al. 2010). However, these studies also suggested that this expression was either not specifically fear-learning related, or the cause of this expression could not be determined. Further, the pattern of activation seen in these studies did not correspond to the tight cluster of neurons we see along the border of the LAvl, but was more randomly distributed throughout the LA. However, analysis of the study of ERK/MAP kinase expression in LA following fear conditioning (Schafe and LeDoux 2000) suggests that similar populations of neurons may show FTL expression and MAP kinase, as previously discussed (Wilson and Murphy 2009). Other studies show that inducing CREB expression in neurons in LA renders these neurons more likely to be involved in fear-memory formation (Han et al. 2007, 2009). Selectively deleting these neurons after learning blocked expression of that fear memory, which shows that the same cells involved in memory formation are involved in memory recall (Han et al. 2009). It would be interesting to determine the CREB status of the neurons that we identified in LAvl following conditioning.

There is extensive evidence that suggests that the LA plays a major role in the formation of CS-US associations in fear conditioning (Pape and Stork 2003; Maren and Quirk 2004; Rodrigues et al. 2004; Kim and Jung 2006). These associations are thought

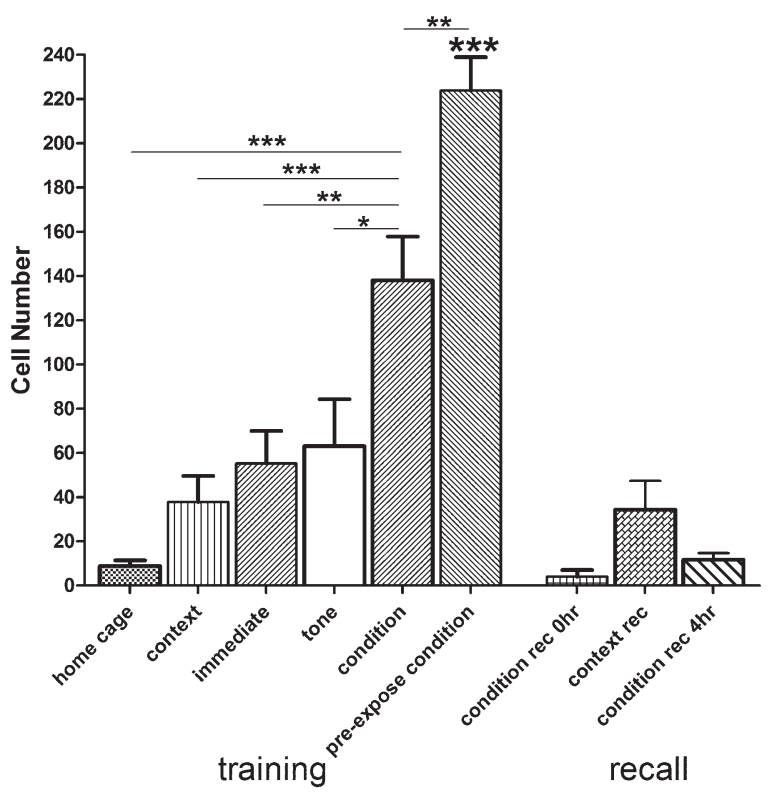

Figure 4. Numbers of $\mathrm{FTL}^{+}$neurons in lateral region of LAvl following fear conditioning and recall. Numbers of $\mathrm{FTL}^{+}$neurons were determined for each group as described in Materials and Methods and are shown as mean \pm SEM. (Condition) Conditioned; (rec) recall. $\left(^{*}\right) P<0.05$; (**) $P<0.01 ;\left({ }^{* *}\right) P<0.001$. (***) The pre-expose conditioned group is significantly different from all nonconditioned groups: $P<0.001$. 

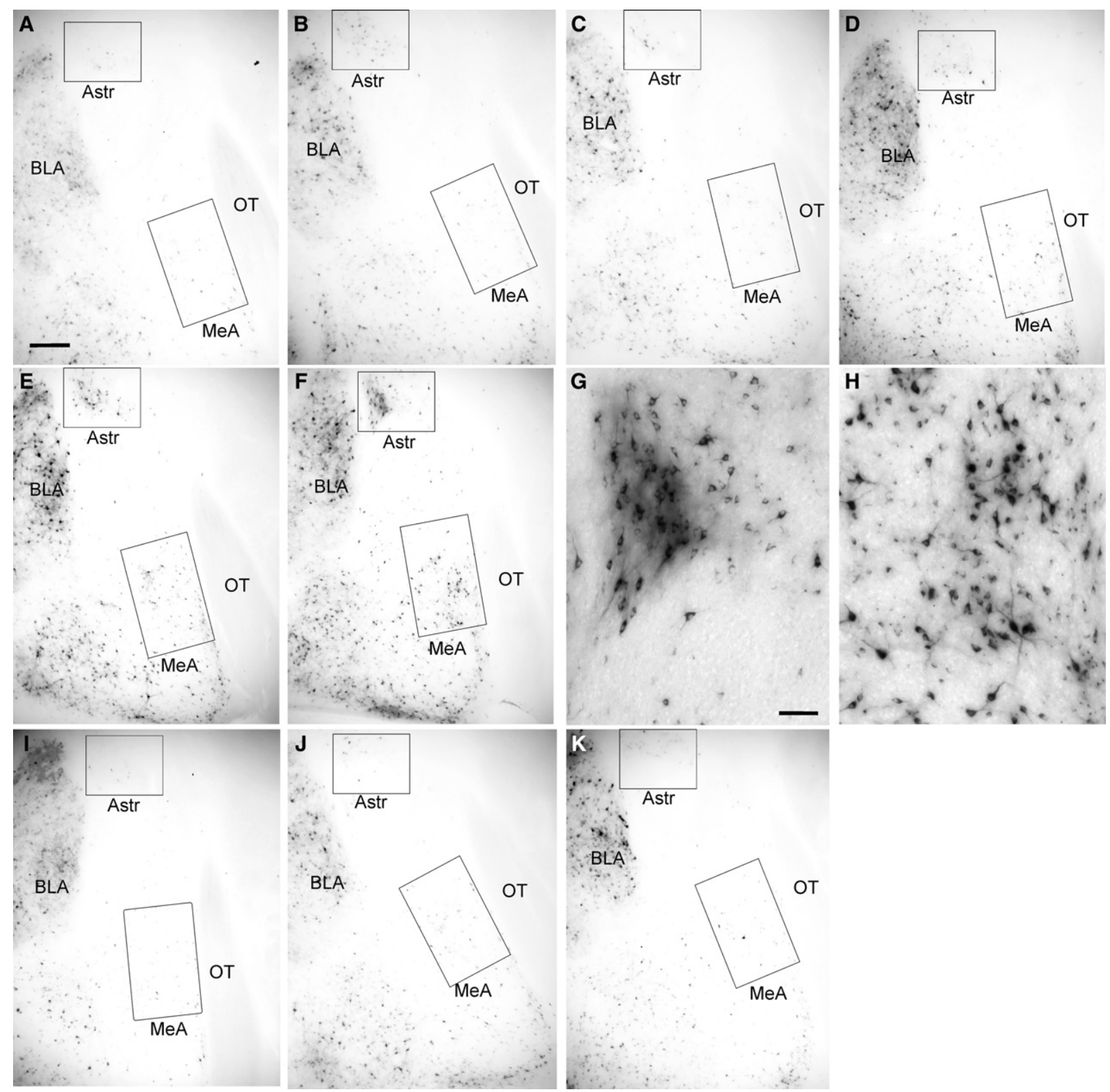

Figure 5. FTL expression in the amygdalo-striatal transition area and MeA following fear conditioning and fear recall. Images of the anterior region of amygdala are shown for $F T L$ mice from the following training groups: $(A)$ home cage, $(B)$ context, $(C)$ immediate shock, $(D)$ tone, $(E)$ conditioned, $(F)$ pre-expose conditioned. Amygdala subnuclei are indicated, including the location of AStr and MeA (Franklin and Paxinos 2007). The indicated rectangles for AStr and MeA show the areas in which $\mathrm{FTL}^{+}$cells were counted for each group. High-power views are shown of $F T L^{+}$neurons within the area of the AStr $(G)$ and $\mathrm{MeA}(H)$, which were counted from a pre-expose conditioned mouse. Images of anterior amygdala are shown from the following recall groups: $(I)$ conditioned recall $0 \mathrm{~h},(J)$ context recall, and $(K)$ conditioned recall $4 \mathrm{~h}$. (Astr) Amygdalo-striatal transition area; (BLA) basolateral amygdala; (MeA) medial amygdala; (OT) optic tract. Scale bar: $A-G=200 \mu \mathrm{m} ; H, I=50 \mu \mathrm{m}$.

to occur in projection neurons in the LA that receive both CS and US input (Ehrlich et al. 2009). In our previous work, we also showed that the learning-specific $F T L^{+}$population in the LAvl are most likely large excitatory projection neurons (Wilson and Murphy 2009). We thus hypothesized that $F^{+} L^{+}$neurons that we identified may be directly involved in fear learning. The c-fosrelated expression within these neurons following learning may be related to the formation of the CS-US association.

We found no increase in numbers of $\mathrm{FTL}^{+}$neurons in LAvl following recall of fear memory compared with basal levels, indicating that these neurons do not express c-fos following fearmemory recall. Our findings are consistent with studies that find no increase in c-fos or other immediate early gene activation across the LA following recall of fear conditioning (Rosen et al. 1998; Hall et al. 2001) and in related studies that utilized predictive fear learning (Furlong et al. 2010). Others do find c-fos activation across the LA following recall (Scicli et al. 2004). The reason for these different findings is not known, but may be associated with different training and testing conditions between these studies. In any case, the pattern of c-fos activation in LA seen in the study of Scicli et al. (2004) did not correspond to the discrete population of neurons we see in LAvl. In summary, these findings suggest that the discrete population of neurons we identified in LAvl only express c-fos following fear learning. An interpretation of this 

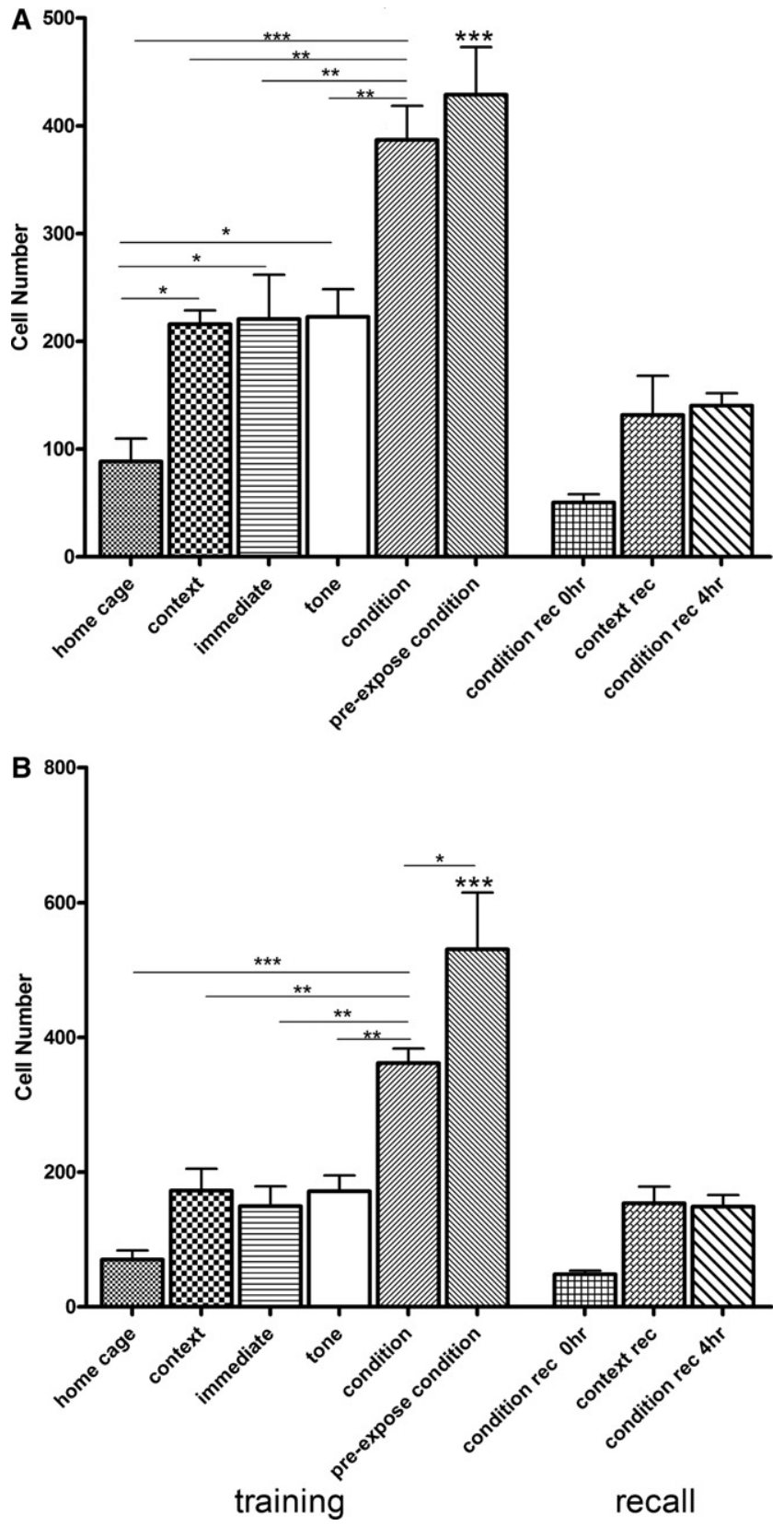

Figure 6. Numbers of $F T L^{+}$neurons in $\operatorname{AStr}(A)$ and $\mathrm{MeA}(B)$ following fear conditioning and recall. Numbers of $F T L^{+}$neurons were determined for each group as described in the Materials and Methods and are shown as mean \pm SEM. (condition) Conditioned; (rec) recall. $\left({ }^{*}\right) P<0.05 ;\left(^{* *}\right)$ $P<0.01 ;\left({ }^{* *}\right) P<0.001$. $\left(^{* *}\right)$ The pre-expose conditioned group is significantly different from all nonconditioned groups: $P<0.001$.

finding is that these neurons undergo plasticity following fear learning associated with the formation of the primary fear memory, but there is no further plasticity following fear-memory recall. If this were the case, these neurons may have altered firing properties that contribute to the conditioned behavior of the mice following recall, but no further changes occur.

Some neurons within basolateral amygdala (BLA) have reported activation following both training and recall (Reijmers et al. 2007). Under our experimental conditions, there may have been conditioned recall-induced activation within BLA, but we were unable to distinguish it from activation due to other stimuli (i.e., in our control groups). In addition, a recent study reported a common pattern of neurons in the dorsal LA following auditory fear conditioning (Bergstrom et al. 2011), but this pattern was not detectable in our context fear-conditioning experiments.

\section{Amygdalo-striatal transition area}

The AStr was the second area we analyzed that showed learningspecific differences in FTL expression. The largest increase in $\mathrm{FTL}^{+}$neurons were found in the conditioned groups of mice compared with all other groups. Part of the expression may also be due to exposure to the novel context. In context, in the immediate shock and tone mice (that are all exposed to a novel context) there were increases in $\mathrm{FTL}^{+}$neurons compared with the home-cage mice. There was no significant increase in FTL expression in any of the recall groups, demonstrating that recall of fear memory has no significant influence on FTL activation in the AStr. Additionally, the conditioned recall group of mice displayed high fear expression, but this appeared to have no influence on FTL expression in the regions we studied.

The learning-specific FTL expression we identified in AStr may reflect changes involved in the formation of memory, such as synaptic change or other uncharacterized cellular changes. The AStr also receives sensory information from the thalamus and olfactory bulbs. It is thus possible that this region could also be a stimulus convergence region for multiple sensory inputs, in addition to receiving inputs from amygdala (Jolkkonen et al. 2001). Alternatively, this expression may be learning related, but indirectly involved with plasticity underlying encoding of memory. For example, there is substantial evidence that amygdala modulates emotional memories via influencing changes in other brain regions (McGaugh 2004).

The LA, as well as the accessory basal nuclei of the amygdala, provide heavy projections to the AStr, and this region is probably a major target of both of these amygdala regions (Jolkkonen et al. 2001). It is possible that the AStr receives direct projections from the learning-specific neurons that we have identified within the LAvl. A substantial output of the AStr includes basal ganglia including striatum, nucleus accumbens, and substantia nigra (Shammah-Lagnado et al. 1999). It has been proposed that emotional stimuli can evoke behavioral responses via these pathways, such as the orienting response (Jolkkonen et al. 2001). Recent studies using unit and local field-potential recordings show coupling of BLA with striatum during learning (Popescu et al. 2009). The different basal ganglia are implicated in regulation of movement and complex motor function (Saint-Cyr et al. 1995). This activation of basal ganglia could thus be involved in the regulation of higher order fear-related behaviors. Where such behaviors have not been studied in our model of fear conditioning and where freezing behavior is a predominant response, they might include fear responses such as avoidance and escape.

The $\mathrm{FTL}^{+}$neurons in the AStr displayed mixed size and morphology, suggesting that different classes of neurons were activated. There were both large and small neurons with FTL activation. These neurons may represent both excitatory as well as inhibitory neurons within the Astr.

In order to determine what role the AStr may have in fear learning and memory, further studies need to be undertaken to examine the function of the $F T L^{+}$AStr neurons and determine the FOS-related changes that occur within these neurons after fear learning. FOS up-regulation implies increased transcription and translation (Knapska et al. 2007); thus, determining which genes are transcribed and translated within AStr following fear conditioning may help us to understand how the AStr neurons are involved. 


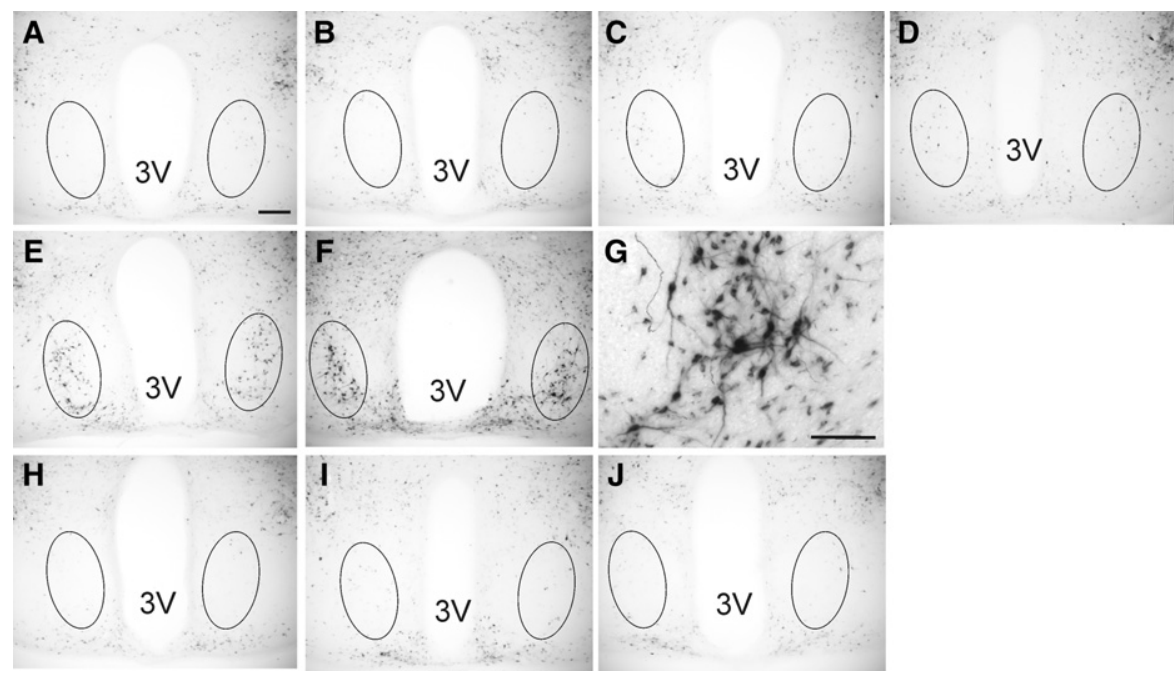

Figure 7. Learning-specific FTL activation in ventromedial hypothalamus. Images of the mid-anterior region of $\mathrm{VMH}$ are shown for $F T L$ mice from the following training groups: $(A)$ home cage, $(B)$ context, $(C)$ immediate shock, $(D)$ tone, $(E)$ conditioned, $(F)$ pre-expose conditioned. $(G)$ A high-power view of $\mathrm{FTL}^{+}$neurons within the area of $\mathrm{VMH}$ that was counted from a pre-expose conditioned mouse. Images of $\mathrm{VMH}$ are shown from the following recall groups: $(H)$ conditioned recall $0 \mathrm{~h},(I)$ context recall, and $(J)$ conditioned recall $4 \mathrm{~h}$. The ellipse indicates the area in which $F T L^{+}$neurons were counted. 3V, third ventricle. Scale bar: $A-F, H-J=200 \mu \mathrm{m} ; G=100 \mu \mathrm{m}$.

\section{Medial amygdala}

The $\mathrm{FTL}^{+}$population of cells identified in the MeA were found in anterior-to-midsections of this region. The cells here were, like in the AStr, of mixed morphology, with large and small neurons. The MeA contains both excitatory and inhibitory neurons (Bian et al. 2008), and the large and small neurons may represent this mixed population. In this area, there was a very clear increase in the number of $\mathrm{FTL}^{+}$neurons in the conditioned group compared with all other groups and a further significant increase in the pre-expose conditioned group. Any other increases over the home-cage baseline were not significant. This pattern of expression suggests a clear activation of neurons specifically associated with fear learning in the MeA.

Other studies have reported increases in FOS in MeA following different types of fear-conditioning training (Pezzone et al. 1992; Milanovic et al. 1998; Radulovic et al. 1998; Rosen et al. 1998; Savonenko et al. 1999), but none of these studies distinguished whether this expression was due to learning or nonlearning experiences of the animals during fear conditioning. Some studies have analyzed c-fos expression in MeA following fear-conditioning recall, showing either no effect (Rosen et al. 1998) or some increase (Pezzone et al. 1992; Campeau et al. 1997). In the study of Pezzone et al. (1992), no quantitative analyses were undertaken, so it is difficult to know how much c-fos activation occurred in MeA following recall and how significant it was. In the study of Campeau et al. (1997), the greatest c-fos expression was seen in MeA in unconditioned mice re-exposed to context, suggesting that the c-fos expression was associated with context exposure rather than recall of fear conditioning. One possibility to explain these different results is that MeA will show increased c-fos expression in response to context exposure to different extents in different experiments. In our experiments, there is a trend for increased FTL expression in all mice exposed to context, although this trend is statistically insignificant.

The MeA has been implicated in processing of olfactory signals implicated in fear (Walker et al. 2005) and social olfactory signals (Brennan and Kendrick 2006). Thus, this region may act as et al. 2007). Our results indicate context fear learning results in specific increases in FOS-related activation in this region. It is possible that this activation is related to undefined olfactory stimuli present during our context fear-conditioning experiments.

Not all experiments support a role for MeA in odor-related fear learning. Injection of either an NMDA or an AMPA/kainate

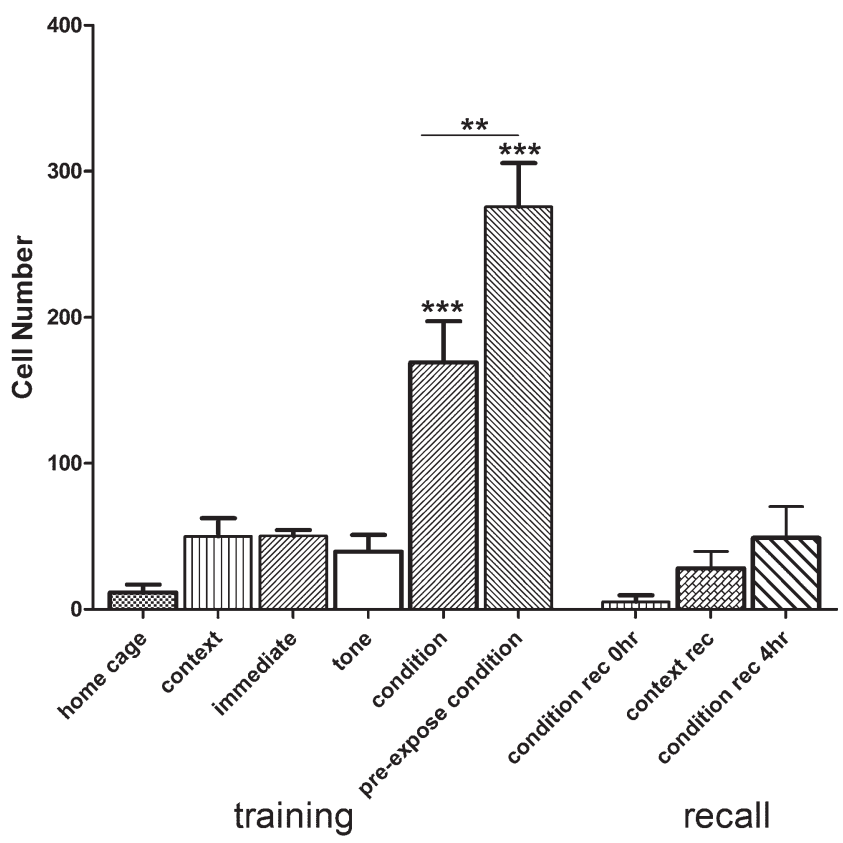

Figure 8. Numbers of $\mathrm{FTL}^{+}$neurons in $\mathrm{VMH}$ following fear conditioning and recall. Numbers of $\mathrm{FTL}^{+}$neurons were determined for each group as described in the Materials and Methods and are shown as mean \pm SEM. (Condition) Conditioned; (rec) recall. (**) $P<0.01$; (***) $P<0.001$, with respect to all nonconditioned groups. 
receptor antagonist into MeA prior to training for context of olfactory fear conditioning did not block fear conditioning (Walker et al. 2005). However, injection of AMPA/kainate receptor antagonist prior to testing did affect fear-potentiated startle. These results were interpreted as the MeA being either an embedded component of the conditioned response pathway, or an indirect modulator of that pathway (Walker et al. 2005). Further studies need to be done in order to determine what the changes in FOS-related expression represent.

\section{Ventromedial hypothalamus}

A population of neurons within the VMH show increased FTL activation only following conditioning, and thus correlate specifically with fear learning. $F T L^{+}$neurons found in this region were generally large, multipolar, and intensely stained. The $\mathrm{VMH}$ is critical for the generation of affective responses to threats (Colpaert 1975). For example, pharmacological blockade of VMH inhibitory circuits with a $\mathrm{GABA}_{\mathrm{A}}$ receptor antagonist enhanced fear behavior (Zagrodzka et al. 2000), suggesting that the VMH contains neurons important for defensive behaviors, and that these are inhibited by GABAergic transmission. Electrical stimulation of VMH produces autonomic and motor responses similar to those induced by natural threats (Lipp and Hunsperger 1978; Lammers et al. 1988).

The VMH is part of a large network of hypothalamic nuclei that are involved in defensive or fear-related behavior (Swanson 2000). Many of the neurons within this network and within VMH show increased FOS expression when rats are exposed to predators or predator odors, which elicit fear expression (Canteras et al. 1997; Dielenberg and McGregor 2001; Staples et al. 2005; Martinez et al. 2008).

In our experiments the VMH shows only low levels of FOSrelated $(F T L)$ expression following fear-memory recall, when the animals show strong fear expression. There is an apparent small increase in FTL activation in VMH following recall, but in the context of our entire study this increase is statistically insignificant, and the predominant increase in activated neurons is only in the conditioned groups. Others have reported increases in c-fos expression in $\mathrm{VMH}$ following recall of conditioned fear to a cue (Campeau et al. 1997) or exposure to a previously shocked context (Beck and Fibiger 1995). However, the levels of induction are relatively low in these studies, broadly similar to our findings. The neurons in VMH are thus not simply activated by fear expression; rather, the activation is fear-learning dependent. Our findings are also consistent with observations that exposure to predator odor not only induces fear, but also induces fear conditioning, and that FOS induction in VMH correlates more with this fear conditioning than with fear expression (Staples et al. 2005). Our findings thus suggest that learning-related changes associated with FOS expression occur in the VMH. These changes may involve synaptic plasticity directly involved with memory formation or other cellular changes that could be directly or indirectly associated with learning. Some experiments suggest that hypothalamic neurons can be involved in learning. For example, lesions of the lateral hypothalamus impair trace learning of flavor associations without interfering with short-delay flavor learning (Touzani and Sclafani 2002). Further, we have found increases in levels of two synaptic proteins, synaptophysin and PSD-95, in hypothalamus following environmental enrichment, a simple learning model (Nithianantharajah et al. 2004).

The major hypothalamic afferents to $\mathrm{VMH}$ are from the amygdala, including both BLA and MeA (Canteras et al. 2001), and both of these also show learning-specific FTL expression. The other learning-specific population of neurons that we identified in AStr also receives heavy projections from BLA. Thus, these four regions are simply interconnected. These four learningspecific populations may form nodes in a circuit for fear conditioning, with each node playing a particular role in the learning process.

\section{Materials and Methods}

\section{Animals}

Mice were male transgenic FTL mice aged between 9 and 11 wk. The mice were housed within the animal facility of the Biomedical Sciences Cluster of the University of Melbourne in a room with a 12-h light-dark cycle. Food and water were supplied ad libitum. One week prior to behavioral experiments, all mice were moved to the behavioral laboratory, where they were housed in quiet $(<60 \mathrm{~dB})$, low light $(15-20$ Lux $)$ conditions. All experimental procedures were adhered to and were approved by the Institutional Animal Care and Use Committee of the University of Melbourne.

\section{Context fear conditioning and recall}

Fear-conditioning experiments were conducted essentially as previously described (Wilson and Murphy 2009). For the training set, mice were subjected to the following treatments. On the training day, home-cage controls were mice taken directly from their home cage with no other treatment and killed. Context control mice were placed in a shock chamber for $3 \mathrm{~min}$. Immediate control mice were placed in the chamber and administered a shock $(0.2 \mathrm{~mA}$ for $3 \mathrm{sec})$ within the first $10 \mathrm{sec}$ of being placed in the chamber, then left in the chamber for another $3 \mathrm{~min}$. Tone control mice were placed in the chamber, and after 3 min they were given a 3-sec 75-dB tone, then left in the chamber for a further $1 \mathrm{~min}$. Conditioned mice were placed in the chamber and after 3 min they were given a 3-sec 0.2-mA shock, then left in the chamber for a further minute. Pre-expose conditioned mice were treated the same way as conditioned mice on the training day; however, $1 \mathrm{~d}$ prior to training, these mice were placed in the shock chamber for $3 \mathrm{~min}$ and then returned to their home cages. Following training, all mice were removed from the shock chamber and returned to their home cages. Four hours following training, all mice were retention tested by being placed back into the shock chamber for $3 \mathrm{~min}$. Upon removal from the chamber following testing, mice were terminally anesthetized and perfused.

For the recall set of treatments, the mice were treated as follows: Conditioned recall mice were conditioned as described above, but given a 1.5-mA shock and tested $4 \mathrm{~d}$ later. Conditioned recall 0 -h mice were removed from the chamber and killed immediately; conditioned recall 4 -h mice were killed $4 \mathrm{~h}$ after removal from the chamber. Context recall mice were treated the same way as context control mice, but were tested $4 \mathrm{~d}$ later and killed $4 \mathrm{~h}$ after testing. The higher shock levels were administered for the recall set of experiments to ensure strong fear responses during testing $4 \mathrm{~d}$ later.

During the training and testing, mice were scored on the following parameters; time spent freezing (Blanchard and Blanchard 1988) and moving, defined as movement of all four paws lateral to the grid floor (Blanchard et al. 1990). For quantification used in behavioral analyses, the following numbers of mice were used: context, $n=5$; immediate, $n=6$; tone, $n=8$; conditioned, $n=9$; pre-expose conditioned, $n=8$; context recall, $n=5$; conditioned recall, $n=9$.

\section{Histochemical procedures}

At the specified times described above, FTL mice were deeply anesthetized with an intraperitoneal overdose $(100 \mu \mathrm{L})$ of Lethabarb (Virbac), then perfused intracardially with $12 \mathrm{~mL}$ of $10 \%$ sucrose in water, followed by $24 \mathrm{~mL}$ of $4 \%$ paraformaldehyde (PFA) containing $0.005 \%$ glutaraldehyde in $0.1 \mathrm{M}$ phosphate buffer $(\mathrm{pH}$ 7.4). After $30 \mathrm{~min}$, brains were dissected out and post-fixed in fresh 4\% PFA for $15 \mathrm{~min}$, washed twice in PBS to remove fixative, cryoprotected in $20 \%$ sucrose/PBS for $48-76 \mathrm{~h}$ at $4^{\circ} \mathrm{C}$, and then 
frozen in OCT (Sakura Finetek) and kept at $-30^{\circ} \mathrm{C}$. Coronal sections $(100 \mu \mathrm{m})$ were cut in a cryostat and placed into the wells of 24 -well tissue culture plates (one to two sections/well) in PBS. For detection of $\beta$-galactosidase activity, PBS was aspirated and sections incubated in assay buffer $\left(20 \mathrm{mM} \mathrm{MgCl}_{2}, 5 \mathrm{mM}\right.$ potassium ferrocyanide, $5 \mathrm{mM}$ potassium ferricyanide, and $1.5 \mathrm{mg} / \mathrm{mL}$ 5-Bromo-4-chloro-3-indolyl- B-D-galactopyranoside [X-gal; Astral]) for $18 \mathrm{~h}$ at room temperature (RT) with agitating. After staining, sections were rinsed in PBS and stored in 4\% PFA until mounted on glass slides. These procedures are more completely described elsewhere (Murphy et al. 2007).

\section{Microscopic examination and data analysis}

Sections were analyzed microscopically and areas which were $\beta$ gal positive were identified by comparison with an atlas of the mouse brain (Franklin and Paxinos 2007). Slides containing serial sections of the brains were scanned using a MIRAX SCAN (Zeiss) and studied using MIRAX viewer software to compare between the levels of activation between training and recall groups within amygdala and hypothalamus. Areas that showed a qualitative difference in the level of activation were then examined quantitatively. Quantitative analysis was undertaken by counting $\mathrm{FTL}^{+}$ cells in matched sections for each group of mice. The counting was performed in an area of the same shape and size for each brain region (see the Results section for further details on which areas were counted).

Cell counts were done using an Olympus microscope (BX61) at a final magnification of $100 \times$. For all areas where neurons were counted, the area of section was examined in detail by focusing throughout its thickness. For LAvl, neurons that were counted were contained within $200 \mu \mathrm{m}$ of the border of the LAvl with the external capsule, were clearly $\mathrm{FTL}^{+}$, and had a stellate morphology with at least two processes. For the AStr and MeA regions, all cells that were clearly identifiable were counted, even cells with relatively weak FTL staining. For the VMH, only the large stained neurons with visible processes within the defined region (see Results) were included in the count. For quantification of all regions, the following numbers of mice were used: home cage, $n=5$; context, $n=4$; immediate, $n=4$; tone, $n=4$; conditioned, $n=5$; pre-expose conditioned, $n=4$; conditioned recall $0 \mathrm{~h}, n=4$; context recall, $n=4$; conditioned recall $4 \mathrm{~h}, n=5$.

Statistical significance was determined using a one way ANOVA, with Tukey's post hoc test for comparison between groups. Prism software (GraphPad Software) was used for performing statistical analysis.

\section{Acknowledgments}

We thank Laura Smith, Ata Ali, and Dania Jailuddin for their involvement in some of the experimental aspects of this work, as well as Marica Kesar and the staff of the Biomedical Animal Services Facility, University of Melbourne.

\section{References}

Asan E, Yilmazer-Hanke DM, Eliava M, Hantsch M, Lesch KP, Schmitt A. 2005. The corticotropin-releasing factor (CRF)-system and monoaminergic afferents in the central amygdala: Investigations in different mouse strains and comparison with the rat. Neuroscience 131: 953-967.

Beck CH, Fibiger HC. 1995. Conditioned fear-induced changes in behavior and in the expression of the immediate early gene $c$-fos: With and without diazepam pretreatment. J Neurosci 15: 709-720.

Bergstrom HC, McDonald CG, Johnson LR. 2011. Pavlovian fear conditioning activates a common pattern of neurons in the lateral amygdala of individual brains. PLoS One 6: e15698. doi: 10.1371/ journal.pone.0015698.

Bian X, Yanagawa Y, Chen WR, Luo M. 2008. Cortical-like functional organization of the pheromone-processing circuits in the medial amygdala. J Neurophysiol 99: 77-86.

Blanchard DC, Blanchard RJ. 1988. Ethoexperimental approaches to the biology of emotion. Annu Rev Psychol 39: 43-68.
Blanchard DC, Blanchard RJ, Tom P, Rodgers RJ. 1990. Diazepam changes risk assessment in an anxiety/defense test battery. Psychopharmacology 101: $511-518$.

Brennan PA, Kendrick KM. 2006. Mammalian social odours: Attraction and individual recognition. Philos Trans R Soc Lond B Biol Sci 361: 2061-2078.

Campeau S, Falls WA, Cullinan WE, Helmreich DL, Davis M, Watson SJ. 1997. Elicitation and reduction of fear: Behavioural and neuroendocrine indices and brain induction of the immediate-early gene $c$-fos. Neuroscience 78: 1087-1104.

Canteras NS, Simerly RB, Swanson LW. 1995. Organization of projections from the medial nucleus of the amygdala: A PHAL study in the rat. J Comp Neurol 360: 213-245.

Canteras NS, Chiavegatto S, Ribeiro do Valle LE, Swanson LW. 1997. Severe reduction of rat defensive behavior to a predator by discrete hypothalamic chemical lesions. Brain Res Bull 44: 297-305.

Canteras NS, Ribeiro-Barbosa ER, Comoli E. 2001. Tracing from the dorsal premammillary nucleus prosencephalic systems involved in the organization of innate fear responses. Neurosci Biobehav Rev 25: 661-668.

Cirelli C, Tononi G. 2000. On the functional significance of $c$-fos induction during the sleep-waking cycle. Sleep 23: 453-469.

Colpaert FC. 1975. The ventromedial hypothalamus and the control of avoidance behavior and aggression: Fear hypothesis versus response-suppression theory of limbic system function. Behav Biol 15: $27-44$.

Day HE, Nebel S, Sasse S, Campeau S. 2005. Inhibition of the central extended amygdala by loud noise and restraint stress. Eur J Neurosci 21: $441-454$.

Day HE, Kryskow EM, Nyhuis TJ, Herlihy L, Campeau S. 2008. Conditioned fear inhibits c-fos mRNA expression in the central extended amygdala. Brain Res 1229: 137-146.

Dielenberg RA, McGregor IS. 2001. Defensive behavior in rats towards predatory odors: A review. Neurosci Biobehav Rev 25: 597-609.

Dong HW, Petrovich GD, Swanson LW. 2001. Topography of projections from amygdala to bed nuclei of the stria terminalis. Brain Res Brain Res Rev 38: 192-246.

Ehrlich I, Humeau Y, Grenier F, Ciocchi S, Herry C, Luthi A. 2009. Amygdala inhibitory circuits and the control of fear memory. Neuron 62: $757-771$

Fanselow MS, LeDoux JE. 1999. Why we think plasticity underlying Pavlovian fear conditioning occurs in the basolateral amygdala. Neuron 23: 229-232.

Fanselow MS, Poulos AM. 2005. The neuroscience of mammalian associative learning. Annu Rev Psychol 56: 207-234.

Franklin KBJ, Paxinos G. 2007. The mouse brain in stereotaxic coordinates, 3rd ed. Academic Press, San Diego, CA.

Furlong TM, Cole S, Hamlin AS, McNally GP. 2010. The role of prefrontal cortex in predictive fear learning. Behav Neurosci 124: 574-586.

Hall J, Thomas KL, Everitt BJ. 2001. Fear memory retrieval induces CREB phosphorylation and Fos expression within the amygdala. Eur $J$ Neurosci 13: 1453-1458.

Han JH, Kushner SA, Yiu AP, Cole CJ, Matynia A, Brown RA, Neve RL, Guzowski JF, Silva AJ, Josselyn SA. 2007. Neuronal competition and selection during memory formation. Science 316: 457-460.

Han JH, Kushner SA, Yiu AP, Hsiang HL, Buch T, Waisman A, Bontempi B, Neve RL, Frankland PW, Josselyn SA. 2009. Selective erasure of a fear memory. Science 323: 1492-1496.

Holahan MR, White NM. 2004. Amygdala c-Fos induction corresponds to unconditioned and conditioned aversive stimuli but not to freezing. Behav Brain Res 152: 109-120.

Jolkkonen E, Pikkarainen M, Kemppainen S, Pitkanen A. 2001. Interconnectivity between the amygdaloid complex and the amygdalostriatal transition area: A PHA-L study in rat. J Comp Neurol 431: $39-58$

Kim JJ, Jung MW. 2006. Neural circuits and mechanisms involved in Pavlovian fear conditioning: A critical review. Neurosci Biobehav Rev 30: 188-202.

Knapska E, Radwanska K, Werka T, Kaczmarek L. 2007. Functional internal complexity of amygdala: Focus on gene activity mapping after behavioral training and drugs of abuse. Physiol Rev 87: 1113-1173.

Kovacs KJ. 2008. Measurement of immediate-early gene activation-c-fos and beyond. J Neuroendocrinol 20: 665-672.

Lammers JH, Kruk MR, Meelis W, van der Poel AM. 1988. Hypothalamic substrates for brain stimulation-induced patterns of locomotion and escape jumps in the rat. Brain Res 449: 294-310.

Lanuza E, Moncho-Bogani J, Ledoux JE. 2008. Unconditioned stimulus pathways to the amygdala: Effects of lesions of the posterior intralaminar thalamus on foot-shock-induced c-Fos expression in the subdivisions of the lateral amygdala. Neuroscience 155: 959-968.

LeDoux JE. 1993. Emotional memory: In search of systems and synapses. Ann N Y Acad Sci 702: 149-157. 
LeDoux JE. 2000. Emotion circuits in the brain. Annu Rev Neurosci 23: $155-184$.

Lipp HP, Hunsperger RW. 1978. Threat, attack and flight elicited by electrical stimulation of the ventromedial hypothalamus of the marmoset monkey Callithrix jacchus. Brain Behav Evol 15: 260-293.

Majak K, Pitkanen A. 2003. Activation of the amygdalo-entorhinal pathway in fear-conditioning in rat. Eur I Neurosci 18: 1652-1659.

Maren S, Quirk GJ. 2004. Neuronal signalling of fear memory. Nat Rev Neurosci 5: 844-852.

Martinez RC, Carvalho-Netto EF, Amaral VC, Nunes-de-Souza RL, Canteras NS. 2008. Investigation of the hypothalamic defensive system in the mouse. Behav Brain Res 192: 185-190.

McDonald AJ. 1998. Cortical pathways to the mammalian amygdala. Prog Neurobiol 55: 257-332.

McGaugh JL. 2004. The amygdala modulates the consolidation of memories of emotionally arousing experiences. Annu Rev Neurosci 27: 1-28.

Milanovic S, Radulovic J, Laban O, Stiedl O, Henn F, Spiess J. 1998. Production of the Fos protein after contextual fear conditioning of C57BL/6N mice. Brain Res 784: 37-47.

Murphy M, Greferath U, Wilson YM. 2007. A method for detecting functional activity related expression in gross brain regions, specific brain nuclei and individual neuronal cell bodies and their projections. Biol Proced Online 9: 1-8.

Nithianantharajah J, Levis H, Murphy M. 2004. Environmental enrichment results in cortical and subcortical changes in levels of synaptophysin and PSD-95 proteins. Neurobiol Learn Mem 81: 200-210

Pape HC, Stork O. 2003. Genes and mechanisms in the amygdala involved in the formation of fear memory. Ann N Y Acad Sci 985: 92-105.

Pare D, Quirk GJ, Ledoux JE. 2004. New vistas on amygdala networks in conditioned fear. J Neurophysiol 92: 1-9.

Pezzone MA, Lee WS, Hoffman GE, Rabin BS. 1992. Induction of c-Fos immunoreactivity in the rat forebrain by conditioned and unconditioned aversive stimuli. Brain Res 597: 41-50.

Pitkanen A, Savander V, LeDoux JE. 1997. Organization of intra-amygdaloid circuitries in the rat: An emerging framework for understanding functions of the amygdala. Trends Neurosci 20: 517-523.

Popescu AT, Popa D, Pare D. 2009. Coherent $\gamma$ oscillations couple the amygdala and striatum during learning. Nat Neurosci 12: 801-807.

Radulovic J, Kammermeier J, Spiess J. 1998. Relationship between Fos production and classical fear conditioning: Effects of novelty, latent inhibition, and unconditioned stimulus preexposure. J Neurosci 18: $7452-7461$.

Radwanska K, Nikolaev E, Knapska E, Kaczmarek L. 2002. Differential response of two subdivisions of lateral amygdala to aversive conditioning as revealed by c-Fos and P-ERK mapping. Neuroreport 13: 2241-2246.

Reijmers LG, Perkins BL, Matsuo N, Mayford M. 2007. Localization of a stable neural correlate of associative memory. Science 317: 1230-1233.

Rodrigues SM, Schafe GE, LeDoux JE. 2004. Molecular mechanisms underlying emotional learning and memory in the lateral amygdala. Neuron 44: $75-91$.

Rosen JB, Fanselow MS, Young SL, Sitcoske M, Maren S. 1998. Immediateearly gene expression in the amygdala following footshock stress and contextual fear conditioning. Brain Res 796: 132-142.
Sah P, Faber ES, Lopez De Armentia M, Power J. 2003. The amygdaloid complex: Anatomy and physiology. Physiol Rev 83: 803-834.

Saint-Cyr JA, Taylor AE, Nicholson K. 1995. Behavior and the basal ganglia. Adv Neurol 65: 1-28.

Savonenko A, Filipkowski RK, Werka T, Zielinski K, Kaczmarek L. 1999. Defensive conditioning-related functional heterogeneity among nuclei of the rat amygdala revealed by c-Fos mapping. Neuroscience $\mathbf{9 4 :}$ $723-733$.

Schafe GE, LeDoux JE. 2000. Memory consolidation of auditory pavlovian fear conditioning requires protein synthesis and protein kinase $\mathrm{A}$ in the amygdala. J Neurosci 20: RC96.

Schettino LF, Otto T. 2001. Patterns of Fos expression in the amygdala and ventral perirhinal cortex induced by training in an olfactory fear conditioning paradigm. Behav Neurosci 115: 1257-1272.

Scicli AP, Petrovich GD, Swanson LW, Thompson RF. 2004. Contextual fear conditioning is associated with lateralized expression of the immediate early gene $c$-fos in the central and basolateral amygdalar nuclei. Behav Neurosci 118: 5-14.

Shammah-Lagnado SJ, Alheid GF, Heimer L. 1999. Afferent connections of the interstitial nucleus of the posterior limb of the anterior commissure and adjacent amygdalostriatal transition area in the rat. Neuroscience 94: $1097-1123$.

Staples LG, Hunt GE, Cornish JL, McGregor IS. 2005. Neural activation during cat odor-induced conditioned fear and 'trial 2 ' fear in rats. Neurosci Biobehav Rev 29: 1265-1277.

Swanson LW. 2000. Cerebral hemisphere regulation of motivated behavior. Brain Res 886: 113-164.

Takahashi LK, Hubbard DT, Lee I, Dar Y, Sipes SM. 2007. Predator odor-induced conditioned fear involves the basolateral and medial amygdala. Behav Neurosci 121: 100-110.

Touzani K, Sclafani A. 2002. Lateral hypothalamic lesions impair flavour-nutrient and flavour-toxin trace learning in rats. Eur J Neurosci 16: $2425-2433$.

Usunoff KG, Schmitt O, Itzev DE, Haas SJ, Lazarov NE, Rolfs A, Wree A. 2009. Efferent projections of the anterior and posterodorsal regions of the medial nucleus of the amygdala in the mouse. Cells Tissues Organs 190: $256-285$

Walker DL, Paschall GY, Davis M. 2005. Glutamate receptor antagonist infusions into the basolateral and medial amygdala reveal differential contributions to olfactory vs. context fear conditioning and expression. Learn Mem 12: 120-129.

Wilson YM, Murphy M. 2009. A discrete population of neurons in the lateral amygdala is specifically activated by contextual fear conditioning. Learn Mem 16: 357-361.

Wilson Y, Nag N, Davern P, Oldfield BJ, McKinley MJ, Greferath U, Murphy M. 2002. Visualization of functionally activated circuitry in the brain. Proc Natl Acad Sci 99: 3252-3257.

Zagrodzka J, Romaniuk A, Wieczorek M, Boguszewski P. 2000. Bicuculline administration into ventromedial hypothalamus: Effects on fear and regional brain monoamines and GABA concentrations in rats. Acta Neurobiol Exp (Wars) 60: 333-343.

Received June 15, 2011; accepted in revised form August 8, 2011. 


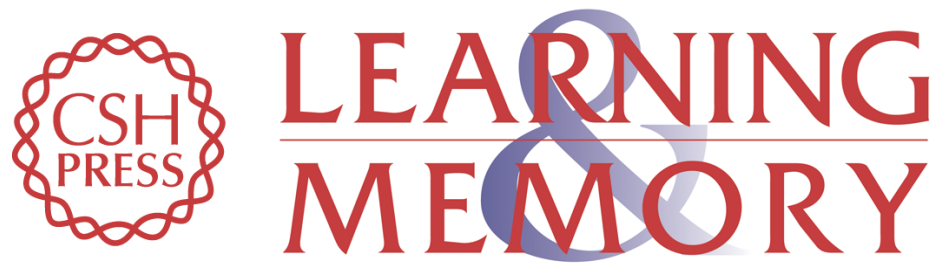

\section{Context fear learning specifically activates distinct populations of neurons in amygdala and hypothalamus}

Lidia Trogrlic, Yvette M. Wilson, Andrew G. Newman, et al.

Learn. Mem. 2011, 18:

Access the most recent version at doi:10.1101//m.2314311

References This article cites 68 articles, 9 of which can be accessed free at:

http://learnmem.cshlp.org/content/18/10/678.full.html\#ref-list-1

License

Email Alerting Receive free email alerts when new articles cite this article - sign up in the box at the Service top right corner of the article or click here. 